\section{Kidney Blood Pressure Research}

\title{
Association of Peridialysis Blood Pressure and Its Variability with Cardiovascular Events in Hemodialysis Patients
}

\author{
Ruoxi Liao Jiameng Li Yuqin Xiong Liping Lin Liya Wang Si Sun Baihai Su \\ Department of Nephrology, West China Hospital, Sichuan University, Chengdu, China
}

\section{Key Words}

Hypertension • Hemodialysis • Blood pressure variability • Antihypertensive drug

\begin{abstract}
Background/Aims: Blood pressure variability (BPV) is a novel cardiovascular risk factor for the population undergoing hemodialysis (HD). Methods: We conducted a retrospective cohort study of 526 HD patients. Four short-term peridialysis BPV metrics were analyzed: systolic blood pressure (SBP) change, SBP coefficient of variation (CV), SBP intradialytic average real variability (ARV), and absolute SBP residual. Multi variate analysis with Cox regression models were used to account for the potential confounders. Results: Short-term BPV is found to be affected by age, pre-dialysis SBP, antihypertensive drugs, dialysis time, and vascular access. Calcium-channel blockers (CCBs) were found to be associated with lower BPV than those on non-CCB therapy or no antihypertensive drugs. Patients dialyzed in the morning had a greater absolute SBP change than those dialyzed in the afternoon or evening. Patients using fistulas had a lower BPV than catheters. Higher BPV metrics including SBP CV (unadjusted hazard ratio [HR]: 1.37, 95\% confidence interval [CI] 1.14-1.66, $p=0.001$ ), SBP intradialytic ARV (unadjusted HR: 1.46, 95\% CI: 1.20-1.77, p<0.001), and SBP residual (unadjusted HR: 1.47, 95\% CI: 1.21 $1.79, p<0.001$ ) were associated with a greater risk of cardiovascular events. After complete multivariate adjustment for other potential confounders, the HR remained statistically significant for SBP intradialytic ARV (HR 1.31, 95\% CI: 1.04-1.66, $\mathrm{p}=0.024)$. Conclusion: Peridialytic BPV may be a potential target for improved blood pressure (BP) management in HD patients. Each short-term BPV metric has different advantages and disadvantages and should be applied according to the clinical context and purpose.




\section{Kidney Blood Pressure Research}

\section{Introduction}

Hypertension is the leading preventable risk factor for cardiovascular disease and death. Owing to its impact, even minor refinements in observance parameters can have a potentially huge impact on patient survival [1]. Accumulative evidence suggests that apart from average blood pressure (BP), blood pressure variability (BPV), which is a measure of BP fluctuations over time, is associated with cardiovascular outcomes in general hypertensive patients [2-6]. It is differentiated into short-term (within 24 hours), mid-term (day-by-day), and long-term (visit-to-visit) BPV based on the time interval [7].

Hemodialysis (HD) patients have a 10- to 20 -fold increased risk for cardiovascular diseases than general population [8]. Part of the reason could be the dialysis procedure that creates a stress on the cardiovascular system and thereby leading to progressive organ damage. Clinical experience dictates that HD patients are particularly prone to increased BPV as denoted by ambulatory blood pressure monitoring (ABPM) [9], suggesting that BPV may contribute to their burden of cardiovascular disease. Toward this direction, several studies exploring the relationship between BPV and cardiovascular outcomes in HD patients have been reported. Most studies focused on long-term BPV and demonstrated the association between long-term BPV and cardiovascular disease [10] and mortality [11,12]. Short-term BPV is less studied and lacks a proper clinically relevant measurement. Flythe et al. defined a measure for intradialytic short-term BPV using absolute regression residuals from a mixed-effect model and suggested that greater short-term BPV could be a predictor of cardiovascular and all-cause mortality in HD patients [13]. However, the short-term BPV metric used in their study was too complex to be implemented in clinical practice.

The intermittent nature of HD is believed to play an important role in the fluctuation of BP. Besides, researchers have found out many other factors associated with BPV, including age, dialytic sessions, and dialytic frequency [14-18].

Further, the effect of the antihypertensive drug class on BPV is not yet understood. While a French study concluded calcium-channel blockers (CCBs) to be better for controlling BPV, a Japanese study conducted on peritoneal dialysis patients showed angiotensin receptor blockers (ARBs) to be better in controlling BPV $[19,20]$.

No clear metric with superior predictive power and operational threshold for peridialytic short-term BPV is available to assess cardiovascular risk and BP management in HD patients. Hence, we conducted a retrospective cohort study in Chinese HD patients to assess four different peridialytic short-term BPV metrics and their association with cardiovascular outcomes in HD patients.

\section{Materials and Methods}

\section{Study design and population}

We performed a retrospective cohort study of patients who commenced maintenance hemodialysis (MHD) between January 1. 2014 and December 31, 2014 at the Dialysis Center of West China Hospital. Renal failure patients aged $\geq 18$ years undergoing MHD in-center on a twice- or thrice-weekly basis for at least 3 months were included in the study. Patients were excluded if they were on dialysis for $<2$ or $>8$ hours, if they received a renal transplant, or if their BP measurement data were not available. Written consent was obtained from all patients. Patients were monitored until December 31,2017, the closing date of the study, or until their death, whichever is earlier, and were withdrawn from the study at the time of kidney transplantation. The study was performed in accordance with the principles of the Declaration of Helsinki and was approved by the Ethics Committee of the West China Hospital.

Data collection

Demographic characteristics (e.g., age, gender, height, body mass index, dialysis vintage, and vascular access type), comorbidities (diabetes, hypertension, heart failure, and coronary artery disease), and a 


\section{Kidney Blood Pressure Research}

complete list of medications with their dosage were obtained from West China Hospital's electronic medical registry. Comorbidities were considered as of cohort entry, and patient compliance toward medication was not assessed.

BP was measured four to six times per dialysis session, with the patient in the seated position using automated BP cuff just before ("pre-dialysis"), immediately after ("post-dialysis"), and during (typically at 1-hour intervals) dialysis. SBP measurements were taken during each dialysis session for all patients. All BP data for 2014 were extracted from the electronic medical registry. Mean values of all BP parameters during the first baseline year were considered as patients' baseline BP parameters. Laboratory parameters including hemoglobin, blood glucose, triglycerides (TGs), uric acid, albumin, creatinine, calcium, and parathyroid hormone (PTH) levels were measured at the time of enrolling and then on a bimonthly or trimonthly basis throughout the study period. The mean values over the study period for different laboratory parameters were considered for further analysis.

To find the impact of different classes of antihypertensive drugs on short-term BPV, patients were divided into five treatment groups: (1) CCB group, receiving either CCBs alone or in combination with nonACEI/ARB antihypertensive drugs; (2) ACEI/ARB group, receiving either ACEI/ARB alone or in combination with non-CCB antihypertensive drugs; (3) CCB + ACEI/ARB group, receiving both CCBs and ACEI/ARBs; (4) non-CCB and non-ACEI/ARB group, receiving antihypertensive drugs other than CCBs and ACEI/ARBs; and (5) patients who were not on antihypertensive drugs. A threshold of 6 months in-treatment during a 1-year baseline visit was considered for categorizing patients into different antihypertensive drug groups.

The co-primary outcomes of interest were cardiovascular events and all-cause mortality. The first occurrence of a cardiovascular event such as coronary heart disease, myocardial infarction, or heart failure was documented. All-cause mortality was defined as death due to any cause. Death due to cardiovascular cause alone was also documented.

\section{Definition and assessment of $B P V$}

We analyzed four different metrics of peridialytic short-term BPV in this study: (1) SBP change, which is the mean difference between post-dialysis SBP and pre-dialysis SBP that represents the gross BP fluctuation/change during dialysis; (2) coefficient of variation (CV) of SBP (CV = standard deviation of SBP/mean SBP); (3) intradialytic average real variability (ARV) of SBP, which is obtained averaging the differences in successive BP readings taken during each dialysis session, is a better indicator of temporal

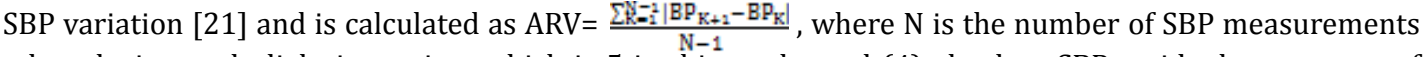
taken during each dialysis session, which is 5 in this study; and (4) absolute SBP residual, a measure of intradialytic SBP variability, which is calculated from a previously described mixed linear model which takes into account the starting SBP, systematic change in SBP during each dialysis session, and random variability in SBP, is calculated as the baseline BP measurements for all the dialysis sessions during 2014, which were incorporated into the mixed effects model to create a predicted pattern of SBP fluctuation during each dialysis session. The absolute difference of this predicted and observed values averaged over treatment sessions and patients is the absolute SBP residual metric [13].

\section{Statistical analysis}

The different baseline characteristics including demographic data were described by means, standard deviations (SD), counts, and proportions as dictated by data type. All BPV metrics were categorized into quartiles. We evaluated the association between baseline characteristics and BPV using ordered logistic regression. Backward selection was applied to identify predictors of BPV in multivariable-adjusted models, by removing variables with $p$-value $\geq 0.1$. We considered two-tailed $p$-values $<0.05$ as statistically significant. Further, Analysis of variance (ANOVA) or Kruskal-Wallis test was done to estimate the effects of certain single factors including age, pre-dialysis SBP, antihypertensive treatments, dialysis time, and vascular access on short-term BPV metrics. Kaplan-Meier estimator was used to calculate the cumulative incidence of cardiovascular events and all-cause death in each quartile group of BPV. Three different Cox regression models were used to calculate hazard ratio (HR) with 95\% confidence interval (CI): (1) unadjusted, (2) partially adjusted (age, gender, mean baseline pre-dialysis SBP), and (3) completely adjusted (age, gender, weight, body mass index [BMI], smoking, dialysis vintage, antihypertensive therapy, vascular access, dialysis frequency, dialysis time, dialysis session length, dialysate calcium concentration, serum creatinine, 


\section{Kidney \\ Blood Pressure Research}

Kidney Blood Press Res 2018;43:1352-1362

\begin{tabular}{l|l}
\hline DOI: 10.1159/000492595 & (C) 2018 The Author(s). Published by S. Karger AG, Basel
\end{tabular}

Published online: 10 August, 2018

www.karger.com/kb

ultrafiltration volume, mean baseline pre-dialysis SBP, mean baseline pre-dialysis diastolic blood pressure (DBP), diabetes, serum albumin, hemoglobin, calcium, and PTH). Variables with $p$-values $<0.1$ and wellestablished predictors were selected as adjustment covariates in multivariate analyses. We confirmed proportionality assumptions of the Cox regression models using Schoenfeld residual. All analyses were performed using STATA 11.0 MP (StataCorp, www.stata.com).

\section{Results}

\section{Study population}

A total of 526 HD patients satisfying the inclusion criteria were enrolled in this study. These patients underwent a total of 59, 412 qualifying HD treatments in the first 12 months of baseline visit, during which 297, 060 SBP measurements were recorded. The various baseline characteristics including demographic details, dialysis parameters, Vascular access, comorbidities, laboratory parameters, and medications are presented in Table 1.

\section{Peridialytic short-term BPV metrics}

The observed results for the four short-term BPV metrics are given in Supplementary Table 1. For all supplemental material see www. karger.com/doi/10.1159/000492595. Mean SBP change was $-6.62 \mathrm{~mm} \mathrm{Hg}$, mean SBP CV was $7.7 \%$, mean SBP intradialytic ARV was $10.83 \mathrm{~mm} \mathrm{Hg}$, and mean absolute SBP residual was $11.02 \mathrm{~mm} \mathrm{Hg}$. Further analysis of influential factors and association with outcomes was facilitated by dividing each metric into four quartiles (Supplementary Table 1).

\section{Factors influencing short-term BPV}

The association between short-term BPV and cohort characteristics determined by logistic regression analysis revealed that the following five cohort parameters influences BPV metrics: (1) age, (2) predialysis BP, (3) antihypertensive drugs, (4) dialysis time, and (5) vascular access. Apart from these, ultrafiltration volume and serum albumin level also influenced BPV (data not shown). The other cohort parameters did not have statistically significant association with BPV.

Age. Older patients tended to have greater BPV. Subjects older than 55 years had greater SBP CV $(0.082 \pm 0.021$ vs. $0.073 \pm 0.018, \mathrm{p}<0.001)$, SBP intradialytic ARV $(11.36 \pm 2.43$ vs. $10.27 \pm 2.48 \mathrm{~mm}$ $\mathrm{Hg}, \mathrm{p}<0.001)$, and SBP residual $(11.52 \pm$ 2.61 vs. $10.51 \pm 2.42 \mathrm{~mm} \mathrm{Hg}, \mathrm{p}<0.001$ ) compared with younger patients (Fig. 1A). Additionally, multifactor logistic regression showed a significant association between age and all four BPV metrics (Table 2).

Table 1. Demographic and clinical parameters of the study population. Continuous variables were shown as mean and SD. ACEI, angiotensin-converting enzyme inhibitor; ARB, angiotensin receptor blocker; CCB, Calcium Channel Blockers; DBP, diastolic blood pressure; ESA, erythropoietin-stimulating agent; SD, Standard Deviation; SBP, systolic blood pressure; PTH, parathyroid hormone

\begin{tabular}{|c|c|}
\hline Parameter & Value \\
\hline Age $(y)$ & $56 \pm 16$ \\
\hline Gender (male:female & $302: 224$ \\
\hline Height $(\mathrm{cm})$ & $162.5 \pm 8.7$ \\
\hline Weight (kg) & $59.5 \pm 11.2$ \\
\hline BMI $\left(\mathrm{kg} / \mathrm{m}^{2}\right)$ & $22.3 \pm 3.2$ \\
\hline Smoking habit & $173(32.9 \%)$ \\
\hline \multicolumn{2}{|l|}{ Dialysis parameters } \\
\hline Dialysis history (y) & $4.6 \pm 3.4$ \\
\hline Interdialytic weight gain/body weight (\%) & $3.8 \pm 1.3$ \\
\hline Dialysis session length (hour) & $3.94 \pm 0.14$ \\
\hline Ultrafiltration volume $(\mathrm{mL})$ & $2233 \pm 781$ \\
\hline $\mathrm{Kt} / \mathrm{V}$ & $1.42 \pm 0.32$ \\
\hline \multicolumn{2}{|l|}{ Vascular access } \\
\hline Fistula & $367(69.8 \%)$ \\
\hline Catheter & $159(30.2 \%)$ \\
\hline Baseline SBP (mm Hg) & $144.2 \pm 17.2$ \\
\hline Baseline DBP (mm Hg) & $80.8 \pm 11.6$ \\
\hline \multicolumn{2}{|l|}{ Complications } \\
\hline Diabetes mellitus & $127(24.1 \%)$ \\
\hline Cardiovascular disease & $50(9.5 \%)$ \\
\hline Hypertension & $389(74.0 \%)$ \\
\hline \multicolumn{2}{|l|}{ Laboratory parameters } \\
\hline Serum albumin $(\mathrm{g} / \mathrm{L})$ & $40.9 \pm 4.3$ \\
\hline Hemoglobin $(\mathrm{g} / \mathrm{L})$ & $101.5 \pm 16.8$ \\
\hline Serum calcium (umol/L) & $2.2 \pm 0.2$ \\
\hline PTH (pmol/L) & $273.1 \pm 224.5$ \\
\hline Serum creatinine (umol/L) & $599.7 \pm 267.8$ \\
\hline \multicolumn{2}{|l|}{ Medications } \\
\hline CCBs & $337(64.1 \%)$ \\
\hline ACEIs/ARBs & $225(42.8 \%)$ \\
\hline Beta-blockers & $237(45.6 \%)$ \\
\hline Alpha-blockers & $91(17.3 \%)$ \\
\hline ESAs & $315(60.0 \%)$ \\
\hline
\end{tabular}




\section{Kidney Bloód Pressure Research}

Fig. 1. Factors affecting BPV. A. Age $(<55$ years vs. $\geq 55$ years). B. Baseline pre-dialysis SBP $\quad(<140$ $\mathrm{mm} \mathrm{Hg}$ vs. $\geq 140 \mathrm{~mm} \mathrm{Hg}$ ). C. Antihypertensive drugs (CCBs, receiving CCBs alone or combined with nonACEI/ARB antihypertensive drugs; ACEI/ARB, receiving ACEI/ARB alone or combined with non-CCB antihypertensive drugs; $\mathrm{CCB}+\mathrm{ACEI} / \mathrm{ARB}$, receiving CCBs and ACEI/ARBs; other, receiving antihypertensive drugs other than CCBs and ACEI/ARBs; none, receiving no antihypertensive drugs) D. CCB classes (nifedipine, amlodipine vs. other CCB classes). E. Dialysis time (morning, afternoon, and evening), F. vascular access (fistula and catheter). SBP, systolic blood pressure; BPV, blood pressure variability; $\mathrm{CV}$, coefficient of variation; ARV, average real variability; CCB calcium channel blocker.

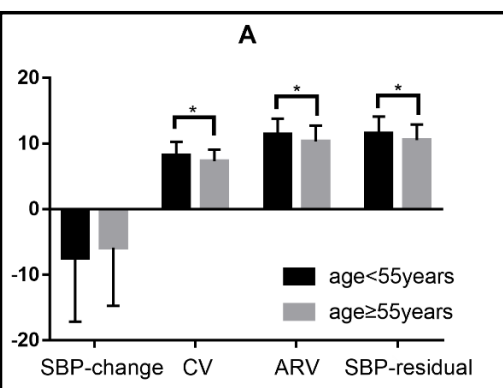

B
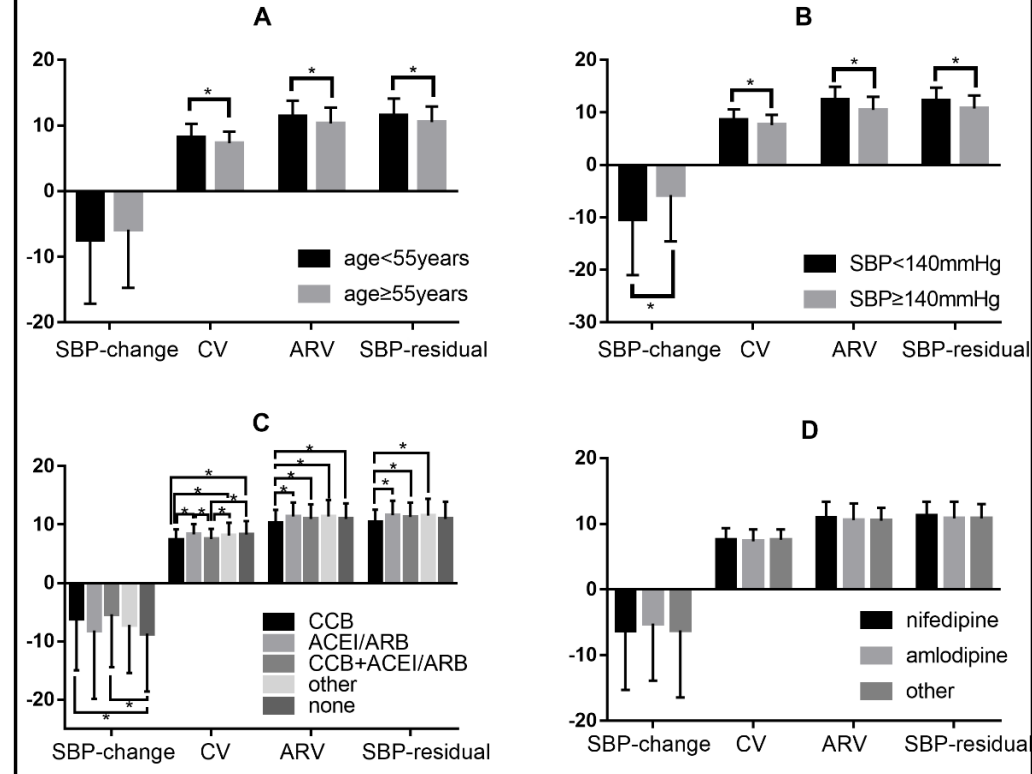

D
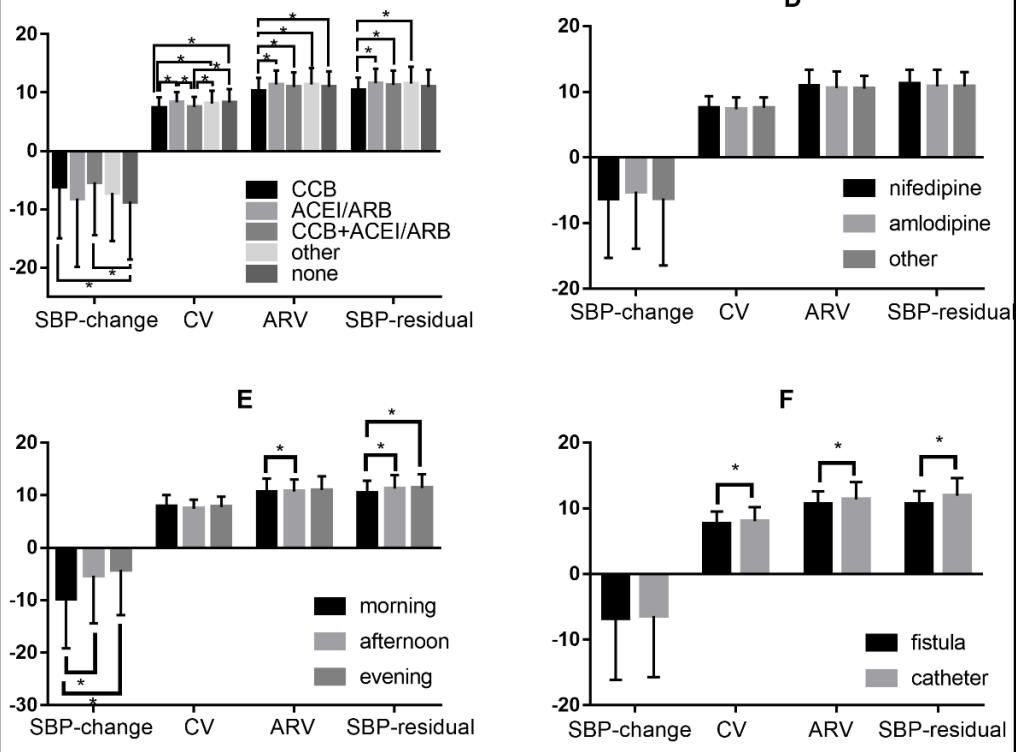

$\mathbf{F}$

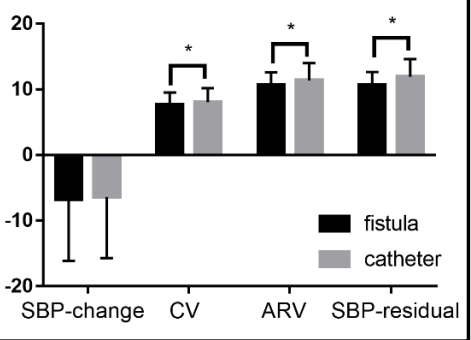

x-axis, BPV metric type; y-axis, SBP, change/mm Hg, CV/\%, ARV/mm Hg, SBP, residual/mm Hg; *p<0.05.

Pre-dialysis SBP. Pre-dialysis SBP also showed a positive correlation with BPV metrics. Patients with pre-dialysis SBP $>140 \mathrm{~mm}$ Hg had greater absolute SBP change $(-10.37 \pm 10.61$ vs. $-5.73 \pm 8.83 \mathrm{~mm} \mathrm{Hg}, \mathrm{p}<0.001)$, SBP CV $(0.085 \pm 0.021$ vs. $0.076 \pm 0.019, \mathrm{p}<0.001)$, SBP intradialytic ARV (12.41 \pm 2.49 vs. $10.45 \pm 2.51 \mathrm{~mm} \mathrm{Hg}, \mathrm{p}<0.001)$, and SBP residual (12.28 \pm 2.44 vs. $10.73 \pm 2.51 \mathrm{~mm} \mathrm{Hg}, \mathrm{p}<0.001$ ) compared with patients with lower pre-dialysis SBP (Fig. 1B). Multifactor logistic regression also suggested pre-dialysis SBP as a significant influencing factor for SBP change (odds ratio [OR]: 0.99, 95\% CI: 0.97-1.00), SBP intradialytic ARV (OR: 1.02, 95\% CI: 1.01-1.04), and SBP residual (OR: 1.03, 95\% CI: 1.02-1.04).

Antihypertensive drugs. This study also revealed drug class-specific effects on BPV independent of pre-dialysis SBP. CCB use was found to be associated with lower SBP CV $(0.07 \pm 0.02$ vs. $0.08 \pm 0.02$ vs. $0.08 \pm 0.02, p=0.002)$, SBP intradialytic ARV $(10.21 \pm 2.31$ vs. $11.38 \pm 2.43$ vs. $11.39 \pm 2.83 \mathrm{~mm} \mathrm{Hg}, \mathrm{p}=0.0498)$, and $\mathrm{SBP}$ residual $(10.39 \pm 2.15$ vs. $11.62 \pm$ 2.48 vs. $11.31 \pm 2.45 \mathrm{~mm} \mathrm{Hg}, \mathrm{p}=0.017$ ) compared with ACEI/ARBs and non-CCB/ACEI/ARB antihypertensive therapy (Fig. 1C). Further analysis on the effects of different CCB classes (nifedipine, amlodipine, and other classes of CCBs) showed no significant difference (Fig. 1D). Multifactor logistic regression suggested an antihypertensive drug class influencing absolute SBP change (Table 2).

Dialysis time. Dialysis time was found to have an impact on BPV independent of baseline pre-dialysis SBP. Patients dialyzed in the morning had a greater absolute SBP change ( -9.75 $\pm 9.39 \mathrm{~mm} \mathrm{Hg})$ than those dialyzed in the afternoon $(-5.31 \pm 9.07 \mathrm{~mm} \mathrm{Hg}, \mathrm{p}<0.001)$ and evening ( $-4.20 \pm 8.63 \mathrm{~mm} \mathrm{Hg}$, p<0.001) (Fig. 1E). However, SBP residual showed an opposite 


\section{Kidney Blood Pressure Research}

Table 2. Factors influencing short-term BPV. ACEI, Angiotensin converting enzyme (ACE) inhibitors; ARB, Angiotensin Receptor Blockers; ARV, average real variability; CCB, Calcium Channel Blockers; CV, coefficient of variation; OR, odds ratio; SBP, systolic blood pressure; 95\% CI, 95\% confidence interval. *Compared with the CCBs group. ${ }^{* *}$ Compared with the morning group. ${ }^{* * *}$ Multifactor analysis was performed by ordered logistic regression, including age, gender, height, weight, body mass index (BMI), smoking, hypertensive drugs, dialysis time, dialysis frequency, dialysate calcium concentration, dialysis session lengths, dialysis vintage, baseline SBP, vascular access, ultrafiltration volume, serum albumin, hemoglobin, calcium, parathyroid hormone (PTH), and equilibrated $\mathrm{Kt} / \mathrm{V}$. "S, single-factor analysis was performed via one-way analysis of variance (ANOVA) or Kruskal-Wallis test according to data distribution and variance equality

\begin{tabular}{|c|c|c|c|c|c|c|c|c|c|c|c|c|c|}
\hline \multirow{3}{*}{\multicolumn{2}{|c|}{ Parameter }} & \multicolumn{3}{|c|}{ Absolute SBP change } & \multicolumn{3}{|c|}{ SBP CV } & \multicolumn{3}{|c|}{ SBP ARV } & \multicolumn{3}{|c|}{ SBP residual } \\
\hline & & \multicolumn{2}{|c|}{ Multifactor ${ }^{* * *}$} & \multirow{2}{*}{$\begin{array}{l}\mathrm{S}^{\prime \prime} \\
\mathrm{p}^{\prime}\end{array}$} & \multicolumn{2}{|c|}{ Multifactor ${ }^{* *+}$} & \multirow{2}{*}{$\begin{array}{l}\mathrm{S}^{*} \\
\mathrm{p}^{\prime}\end{array}$} & \multicolumn{2}{|c|}{ Multifactor } & \multirow{2}{*}{$\begin{array}{l}\mathrm{S}^{\#} \\
\mathrm{p}^{\prime}\end{array}$} & \multicolumn{2}{|l|}{ Multifactor ${ }^{* * *}$} & \multirow{2}{*}{$\begin{array}{l}\mathrm{S}^{\#} \\
\mathrm{p}^{\prime}\end{array}$} \\
\hline & & OR $(95 \% \mathrm{CI})$ & $\mathrm{p}$ & & OR $(95 \% \mathrm{CI})$ & $\mathrm{p}$ & & OR $(95 \% \mathrm{CI})$ & $\mathrm{p}$ & & OR $(95 \% \mathrm{CI})$ & $\mathrm{p}$ & \\
\hline Age & & $\begin{array}{c}0.98 \\
(0.97,1.00)\end{array}$ & 0.010 & 0.060 & $1.03(1.02,1.05)$ & 0.000 & 0.000 & $1.03(1.01,1.05)$ & 0.000 & 0.000 & $1.01(1.00,1.03)$ & 0.005 & 0.000 \\
\hline Pre-dialysis S & & $\begin{array}{c}0.99 \\
(0.97,1.00)\end{array}$ & 0.033 & 0.000 & $1.01(1.00,1.02)$ & 0.203 & 0.000 & $1.02(1.01,1.040)$ & 0.001 & 0.000 & $1.03(1.02,1.04)$ & 0.000 & 0.000 \\
\hline \multirow{4}{*}{$\begin{array}{l}\text { Hypertensive } \\
\text { drugs }\end{array}$} & ACEI/ARBs* & $\begin{array}{c}1.11 \\
(0.39,3.19)\end{array}$ & 0.839 & 0.276 & $2.14(0.79,5.82)$ & 0.134 & 0.016 & $2.13(0.79,5.76$ & 0.134 & 0.037 & $2.70(1.01,7.23)$ & 0.047 & 0.015 \\
\hline & $\begin{array}{c}\text { ACEI/ARBs + } \\
\text { CCBs }^{*}\end{array}$ & $\begin{array}{c}1.27 \\
(0.81,1.98)\end{array}$ & 0.293 & 0.261 & $1.39(0.88,2.18)$ & 0.155 & 0.296 & $2.06(1.30,3.24)$ & 0.002 & 0.007 & $2.07(1.31,3.26)$ & 0.002 & 0.002 \\
\hline & $\begin{array}{c}\text { Non- } \\
\text { ACEI/ARB/CCBs }\end{array}$ & $\begin{array}{c}0.53 \\
(0.23,1.21)\end{array}$ & 0.135 & 0.267 & $2.88(1.28,6.46)$ & 0.010 & 0.029 & $2.56(1.12,5.860)$ & 0.026 & 0.014 & $2.35(0.99,5.60)$ & 0.053 & 0.026 \\
\hline & None $^{*}$ & $\begin{array}{c}0.57 \\
(0.34,0.95)\end{array}$ & 0.030 & 0.015 & $1.98(1.17,3.36)$ & 0.011 & 0.001 & $1.35(0.79,2.28$ & 0.268 & 0.018 & $1.21(0.72,2.04)$ & 0.720 & 0.095 \\
\hline \multirow{2}{*}{ Dialysis time } & Afternoon ${ }^{* *}$ & $\begin{array}{c}2.33 \\
(1.54,3.52)\end{array}$ & 0.000 & 0.000 & $0.82(0.54,1.250)$ & 0.359 & 0.300 & $1.01(0.66,1.52$ & 0.978 & 0.148 & $1.69(1.11,2.55)$ & 0.013 & 0.000 \\
\hline & Evening** & $\begin{array}{c}2.75 \\
(1.57,4.83)\end{array}$ & 0.000 & 0.000 & $0.88(0.51,1.54$ & 0.658 & 0.300 & $1.08(0.62,1.87)$ & 0.796 & 0.148 & $1.86(1.06,3.26)$ & 0.030 & 0.004 \\
\hline \multicolumn{2}{|c|}{ Vascular access } & $1.09(0.69,1.71)$ & 0.720 & 0.697 & $0.80(0.50,1.28$ & 0.353 & 0.043 & $0.70(0.45,0.89)$ & 0.007 & 0.010 & $1.48(0.93,2.34)$ & 0.096 & 0.000 \\
\hline \multicolumn{2}{|c|}{ Interdialytic weight gain } & $0.66(0.30,1.41)$ & 0.283 & 0.000 & $1.18(0.52,2.69)$ & 0.692 & 0.002 & $0.97(0.43,2.22)$ & 0.947 & 0.000 & $0.79(0.36,1.74))$ & 0.564 & 0.003 \\
\hline \multicolumn{2}{|c|}{ Serum albumin } & $1.00(0.95,1.06)$ & 0.874 & 0.910 & $0.99(0.94,1.0$ & 0.589 & 0.005 & $0.96(0.91,1.01)$ & 0.128 & 0.000 & $0.92(0.87,0.97)$ & 0.002 & 0.000 \\
\hline \multicolumn{2}{|c|}{ Serum creatinine } & $\begin{array}{c}1.0000 \\
(0.9990,1.0005)\end{array}$ & 0.526 & 0.728 & $\begin{array}{c}1.0004 \\
(0.9997,1.0063)\end{array}$ & 0.258 & 0.111 & $\begin{array}{c}1.0008 \\
(1.0000,1.0016)\end{array}$ & 0.045 & 0.111 & $\begin{array}{c}1.0009 \\
(1.0001,1.0002)\end{array}$ & 0.020 & 0.671 \\
\hline
\end{tabular}

tendency, lowest in the morning group $(10.39 \pm 2.39 \mathrm{~mm} \mathrm{Hg})$ and higher in the afternoon (11.39 $\pm 2.61 \mathrm{~mm} \mathrm{Hg}, \mathrm{p}<0.001)$ and evening group $(11.29 \pm 2.57 \mathrm{~mm} \mathrm{Hg}, \mathrm{p}=0.002)$ (Fig. 1E). Multifactor logistic regression also showed a significant association between dialysis time and SBP change (Table 2).

Vascular access. Subjects dialyzed via arteriovenous fistulas showed a lower BPV than dialyzed with catheters with respect to SBP CV ( $0.07 \pm 0.02$ vs. $0.08 \pm 0.02, p=0.02)$, SBP intradialytic ARV (10.61 \pm 2.42 vs. $11.34 \pm 2.66 \mathrm{~mm} \mathrm{Hg}, \mathrm{p}=0.01)$, and SBP residual (10.64 \pm 2.41 vs. $11.91 \pm 2.71 \mathrm{~mm} \mathrm{Hg}, \mathrm{p}=0.00$ ) (Fig. $1 \mathrm{~F}$ ). Multifactor logistic regression also showed a significant association between vascular access and SBP intradialytic ARV (OR: 0.70, 95\% CI: 0.45-0.89).

\section{Short-term BPV and risk of cardiovascular events and all-cause mortality}

During the study period, 27 incidents of death and 92 cardiovascular events were recorded. Cardiovascular disease was the predominant cause of death $(40.7 \%)$, followed by infections (37.0\%) and stroke (14.8\%). SBP CV (unadjusted HR: 1.37, 95\% CI: 1.14-1.66, $\mathrm{p}=0.001$ ), SBP intradialytic ARV (unadjusted HR: 1.46, 95\% CI: 1.20-1.77, $\mathrm{p}<0.001$ ), and SBP residual (unadjusted HR: 1.47, 95\% CI: 1.21-1.79, p<0.001), which are BPV metrics of higher order, were associated with a greater risk of cardiovascular events (Supplementary Table 2). After partial and complete adjustment of other potential confounders, the HR was diminished, but remained statistically significant for SBP intradialytic ARV (HR: 1.31, 95\% CI: 1.04-1.66, $\mathrm{p}=0.024$ ). All the four short-term BPV metrics showed no significant impacts on all-cause mortality. Statistically significant difference was observed between the different quartiles of short-term BPV metrics (except SBP change) and their association with cardiovascular events (Fig. 2A-2D). SBP intradialytic ARV Q3 and Q4 had a more linear relationship with cardiovascular events, suggesting the scope of having an SBP intradialytic ARV cutoff for clinical risk assessment. Unadjusted, partially adjusted and completely adjusted HRs for cardiovascular events and all-cause death were shown in Fig. 2E-2F and Supplementary Table 2. 


\section{Kidney \\ Blood Pressure \\ Research}

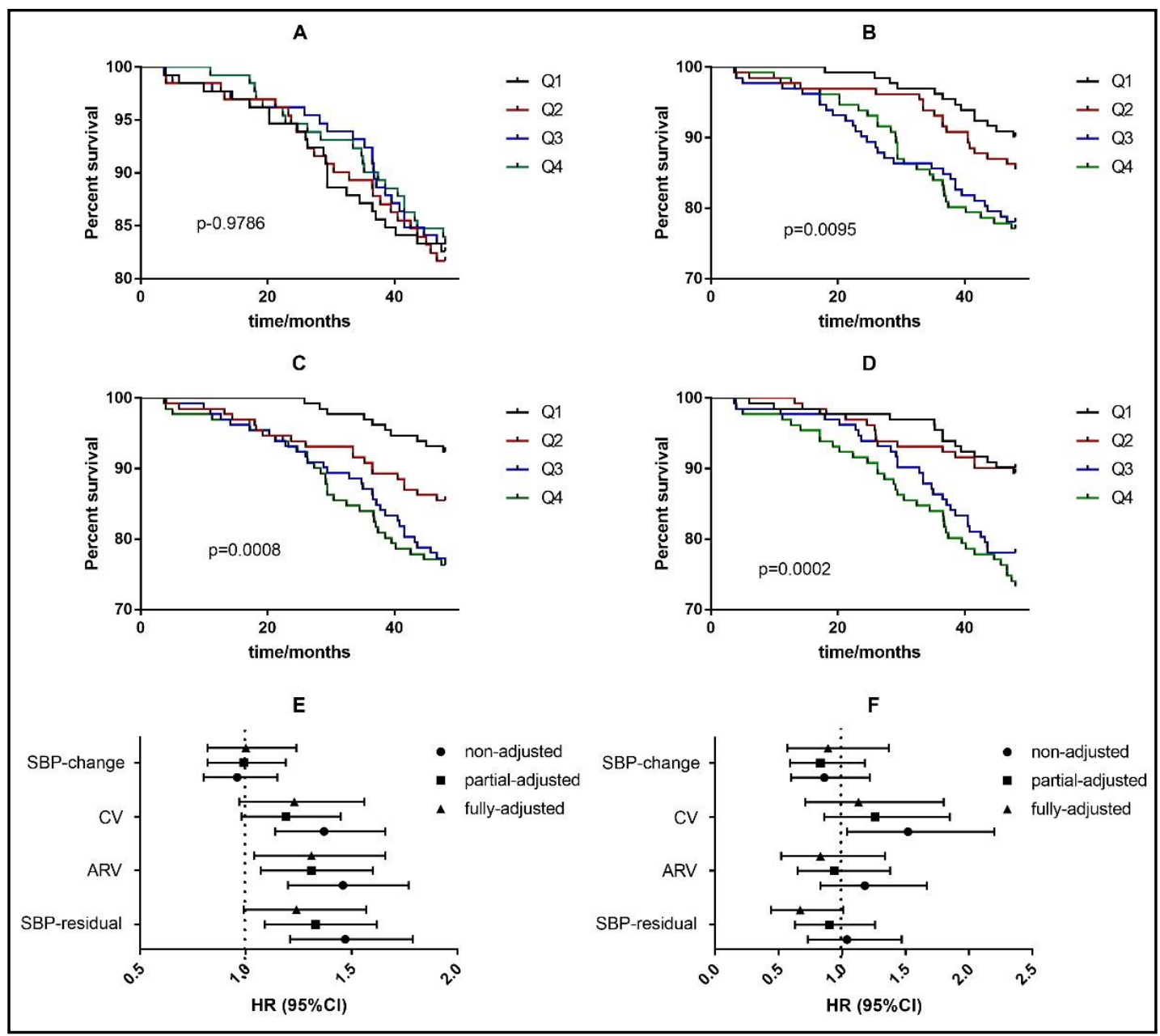

Fig. 2. Cumulative rate of cardiovascular events to quartiles of BPV, HR for cardiovascular events and allcause death. A. SBP change; B. SBP CV; C. SBP ARV; D. SBP residual; E. HR for cardiovascular events; F. HR for all-cause death. SBP, systolic blood pressure; CV, coefficient of variation; ARV, average real variability; HR, hazard ratio; $\mathrm{CI}$, confidence interval; $\mathrm{Q}$ quartile.

\section{Sensitivity analysis}

BPV metrics analyzed as tertiles had a similar association with outcomes as the primary analysis. Analysis with antihypertensive drugs grouped as CCBs, non-CCB-antihypertensive drugs, and non-antihypertensive drug group also revealed similar findings (data not shown).

\section{Discussion}

In this retrospective cohort study, we analyzed four peridialytic short-term BPV metrics and their association with cardiovascular events in HD patients. Age, pre-dialysis SBP, antihypertensive drugs, dialysis time, and vascular access were found to affect the four BPV metrics disparately. SBP intradialytic ARV, which is a more reliable BPV metric, was associated with a greater risk of cardiovascular events and all-cause mortality.

Age is found to be associated with all the four BPV metrics, with age $>55$ years associated with high BPV, except the SBP change. The physiological factors associated with aging such as impairment of cardiovascular autonomic may influence the observed association [22]. 


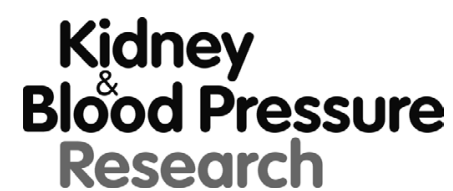

\begin{tabular}{l|l}
\hline Kidney Blood Press Res 2018;43:1352-1362 \\
\hline DOI: 10.1159/000492595 & $\begin{array}{l}\text { (c) 2018 The Author(s). Published by S. Karger AG, Basel } \\
\text { www.karger.com/kbr }\end{array}$ \\
\hline Published online: 10 August, 2018
\end{tabular}

Pre-dialysis SBP, which is a parameter for clinical decision-making in the management of hypertension, was found to be associated with all the four BPV metrics, substantiating its usefulness as a primary metric for hypertension in HD patients, which was earlier endorsed by Shafi et al., 2014.

The antihypertensive drug class was found to have an effect on BPV metrics, with CCBs being the most effective in controlling the short-term BPV. Similar findings were also made in previous studies with long-term BPV in general population [23, 24]. The observed effects of CCBs in controlling short-term BPV might be due to their vasodilator effects [25] and long half-life [26] as well as the sustained release of CCBs [27]. Further, a previous in vivo experimental study showed a low removal of nifedipine by HD, with a dialyzer extraction ratio of $2.3 \% \pm 0.8 \%$ [28], which could also contribute to the better therapeutic outcome with CCBs in HD patients. Intra-class variability of CCB drugs in alleviating short-term variability in BP was observed in a previous randomized crossover study [29], which was in contrary to the current study.

It is the first study that showed the association between dialysis time and BPV. Interestingly, morning dialysis showed a higher absolute SBP change but a lower SBP residual. This difference may be attributed to the fact that SBP residual emphasizes on the deviation from a predicted value, whereas SBP change highlights the extreme values. Further experimental studies are needed to determine which time is best for dialysis.

Vascular access also influences short-term BPV metrics, with fistulas performing better in terms of controlling short-term BPV in our study, which is in accordance to a recent study on long-term BPV [12]. It could be due to the greater influence of catheters on hemodynamics than fistulas, implying fistulas to be favorable for vascular access in HD patients.

DBP variability has also been implicated in predicting cardiovascular outcomes in previous studies [30]. However, no such association was found in the current study (data not shown).

Present guidelines for hypertension focuses on average BP which is not constant but fluctuates from time to time, more so in HD patients. In this context, additional metrics specific for HD patients are required. BPV, which describes fluctuation of BP at different levels, may serve as a better indicator of cardiovascular functioning in HD patients. Previous studies have demonstrated both short- and long-term BPV associated with cardiovascular and all-cause mortality in HD patients $[11,13]$. In this study, higher short-term BPV was associated with a greater risk of cardiovascular events, which substantiates its importance as a potential marker for cardiovascular health in HD patients.

We compared four BPV metrics, namely, SBP change, SBP CV, SBP intradialytic ARV, and SBP residual. SBP change was least associated with the primary outcomes although a previous retrospective cohort study showed a U-shaped association between SBP change and all-cause mortality, with peak survival at $-14 \mathrm{~mm} \mathrm{Hg}$ [31]. We recommend SBP change to be used as a preliminary measure when intradialytic BP measurements are not available.

Since SBP SD increases with SBP, we chose SBP CV instead of SD as the second metric of interest. On this study, SBP CV is the only short-term metric independent of baseline predialysis SBP and is found to be moderately associated with cardiovascular event risk. Since it is not dependent on pre-dialysis SBP, we suggest this metric be applied when baseline SBP differentiates greatly.

SBP intradialytic ARV, which considers the order of measurements, is the most effective marker for cardiovascular events. As SBP changes from time to time during dialysis sessions, SBP intradialytic ARV may best reflect the absolute fluctuation of SBP during HD. Since BP fluctuations lead to alternating periods of myocardial hypoxia, SBP intradialytic ARV thus becomes a potential predictor of cardiovascular events. Hence, we recommend it as a preferred measure for BP management in HD patients. In this study, the number of SBP measurements used to calculate SBP intradialytic ARV was 5. In a previous study, 48 SBP measurements were recommended for accurate determination of ARV in ABPM without compromising its prognostic value [32]. However, owing to the practical difficulties involved 


\section{Kidney \\ Blood Pressure Research}

with measuring multiple SBP measurements during dialysis sessions, an optimal value for a minimum number of readings for intradialytic ARV may be arrived at in future. SBP residual derived from generalized models is also a good outcome marker. In the study conducted by Flythe et al., SBP residual is associated with increased all-cause (HR :1.26, 95\% CI: 1.081.47) and cardiovascular mortality (HR: 1.32, 95\% CI: 1.01-1.72) [13]. However, this metric requires complex analytical approaches, making it less practical for clinical use. Besides, as it relies on BP paradigms, it may fail when there is a paradigm shift.

To the best of our knowledge, this is the first cohort study to analyze different shortterm BPV metrics in HD patients, giving clinicians options to choose BPV parameters based on the clinical context and data availability. Further, it is the largest cohort study on BPV in Chinese HD population with the largest intradialytic BP database, giving a picture of BPV in Asian population.

We have to admit that there are still some limitations. First, as with all retrospective cohort studies, bias may exist. We have adjusted confounding variables when evaluating BPV and cardiovascular events, but we cannot dismiss the potential for missing confounders. Second, we evaluated the effects of antihypertensive drugs, but we were unable to assess compliance. Third, as we included patients from 2014, the follow-up time may be too short to estimate the difference in death events according to different BPV quartiles. So, further prospective, randomized, controlled trials with a long follow-up and a large sample size are needed. Future studies aimed at establishing an operational threshold for intradialytic SBP ARV will go a long way in the management of cardiovascular events in HD patients.

\section{Conclusion}

This study demonstrated the association of short-term BPV with cardiovascular events, with SBP intradialytic ARV as the best predictor for cardiovascular events, suggesting that it could be a potential marker for amelioration and management of BP in HD patients.

\section{Acknowledgements}

This work was financially sponsored by the National Natural Science Foundation of China (No. 51433007-1) and the State Key Research Development Programme of China (2016YFC1103004).

We gratefully acknowledge all the clinicians, statisticians, and laboratory technicians who contributed to the study. We acknowledge Dr G. Kaushik Subramanian, Indegene, Bangalore, India, for English editing and revising.

R.L. was responsible for the study procedure, drafting, and revising the manuscript. J.L. and Y.X. performed data collection. L.L. managed study procedures and data collection. L.W. conducted statistical analysis. B.S. performed data analysis and interpretation.

\section{Disclosure Statement}

There are no conflicts of interest. 


\section{Kidney \\ Blood Pressure Research}

\section{References}

-1 Mancia G, Fagard R, Narkiewicz K, Redon J, Zanchetti A, Böhm M, Christiaens T, Cifkova R, De Backer G, Dominiczak A, Galderisi M, Grobbee DE, Jaarsma T, Kirchhof P, Kjeldsen SE, Laurent S, Manolis AJ, Nilsson PM, Ruilope LM, Schmieder RE, et al.: 2013 ESH/ESC Guidelines for the management of arterial hypertension: The Task Force for the management of arterial hypertension of the European Society of Hypertension (ESH) and of the European Society of Cardiology (ESC). Eur Heart J 2013;34:2159-2219.

-2 Mancia G, Schumacher H, Böhm M, Redon J, Schmieder RE, Verdecchia P, Sleight P, Teo K, Yusuf S: Relative and Combined Prognostic Importance of On-Treatment Mean and Visit-to-Visit Blood Pressure Variability in ONTARGET and TRANSCEND Patients. Hypertension 2017;70:938-948.

-3 Ohkuma T, Woodward M, Jun M, Muntner P, Hata J, Colagiuri S, Harrap S, Mancia G, Poulter N, Williams B, Rothwell P, Chalmers J, ADVANCE Collaborative Group: Prognostic Value of Variability in Systolic Blood Pressure Related to Vascular Events and Premature Death in Type 2 Diabetes Mellitus: The ADVANCE-ON Study. Hypertension 2017;70:461-468.

4 Oishi E, Ohara T, Sakata S, Fukuhara M, Hata J, Yoshida D, Shibata M, Ohtsubo T, Kitazono T, Kiyohara Y, Ninomiya T: Day-to-Day Blood Pressure Variability and Risk of Dementia in a General Japanese Elderly Population: The Hisayama Study. Circulation 2017;136:516-525.

-5 Proietti M, Romiti GF, Olshansky B, Lip GYH: Systolic Blood Pressure Visit-to-Visit Variability and Major Adverse Outcomes in Atrial Fibrillation Novelty and Significance: The AFFIRM Study (Atrial Fibrillation Follow-Up Investigation of Rhythm Management). Hypertension 2017;70:949-958.

-6 Tai C, Sun Y, Dai N, Xu D, Chen W, Wang J, Protogerou A, van Sloten TT, Blacher J, Safar ME, Zhang Y, Xu Y: Prognostic significance of visit-to-visit systolic blood pressure variability: a meta-analysis of 77, 299 patients. J Clin Hypertens (Greenwich) 2015;17:107-115.

-7 Stergiou GS, Parati G, Vlachopoulos C, Achimastos A, Andreadis E, Asmar R, Avolio A, Benetos A, Bilo G, Boubouchairopoulou N, Boutouyrie P, Castiglioni P, de la Sierra A, Dolan E, Head G, Imai Y, Kario K, Kollias A, Kotsis V, Manios E, et al.: Methodology and technology for peripheral and central blood pressure and blood pressure variability measurement: current status and future directions - Position statement of the European Society of Hypertension Working Group on blood pressure monitoring and cardiovascular variability. J Hypertens 2016;34:1665-1677.

8 Weiner DE, Tighiouart H, Amin MG, Stark PC, MacLeod B, Griffith JL, Salem DN, Levey AS, Sarnak MJ: Chronic kidney disease as a risk factor for cardiovascular disease and all-cause mortality: a pooled analysis of community-based studies. J Am Soc Nephrol 2004;15:1307-1315.

9 Muntner P, Shimbo D, Tonelli M, Reynolds K, Arnett DK, Oparil S: The relationship between visit-to-visit variability in systolic blood pressure and all-cause mortality in the general population: findings from NHANES III, 1988 to 1994. Hypertension 2011;57:160-166.

-10 Norioka N, Iwata S, Yanagi S, Ishii H, Sugioka K, Kamimori K, Ota T, Di Tullio MR, Homma S, Yoshiyama M: Higher visit-to-visit intra-dialytic blood pressure is associated with the progression of aortic valve stenosis in chronic hemodialysis patients. Heart Vessels 2016;31:1491-1496.

-11 Shafi T, Sozio SM, Bandeen-Roche KJ, Ephraim PL, Luly JR, St Peter WL, McDermott A, Scialla JJ, Crews DC, Tangri N, Miskulin DC, Michels WM, Jaar BG, Herzog CA, Zager PG, Meyer KB, Wu AW, Boulware LE, DEcIDE Network Patient Outcomes in End Stage Renal Disease Study Investigators: Predialysis systolic BP variability and outcomes in hemodialysis patients. J Am Soc Nephrol 2014;25:799-809.

-12 Sumida K, Molnar MZ, Potukuchi PK, Thomas F, Lu JL, Yamagata K, Kalantar-Zadeh K, Kovesdy CP: Preend-stage renal disease visit-to-visit systolic blood pressure variability and post-end-stage renal disease mortality in incident dialysis patients. J Hypertens 2017;35:1816-1824.

-13 Flythe JE, Inrig JK, Shafi T, Chang TI, Cape K, Dinesh K, Kunaparaju S, Brunelli SM: Association of intradialytic blood pressure variability with increased all-cause and cardiovascular mortality in patients treated with long-term hemodialysis. Am J Kidney Dis 2013;61:966-974.

14 Karpetas A, Loutradis C, Bikos A, Tzanis G, Koutroumpas G, Lazaridis AA, Mavromatidis K, Liakopoulos V, Papagianni A, Zebekakis P, Ruilope LM, Parati G, Sarafidis PA: Blood pressure variability is increasing from the first to the second day of the interdialytic interval in hemodialysis patients. J Hypertens 2017;35:25172526. 


\section{Kidney \\ Blood Pressure Research}

15 Kim HY, Kang YU, Kim CS, Choi JS, Bae EH, Ma SK, Kim SW: Association of age and BP variability with longterm mortality in hemodialysis patients. Kidney Blood Press Res 2013;38:172-180.

16 Murashima M, Kumar D, Doyle AM, Glickman JD: Comparison of intradialytic blood pressure variability between conventional thrice-weekly hemodialysis and short daily hemodialysis. Hemodial Int 2010;14:270-277.

17 Jeffers BW, Zhou D: Relationship Between Visit-to-Visit Blood Pressure Variability (BPV) and Kidney Function in Patients with Hypertension. Kidney Blood Press Res 2017;42:697-707.

$>18$ Li W, Yu Y, Liang D, Jia EZ: Factors Associated with Blood Pressure Variability Based on Ambulatory Blood Pressure Monitoring in Subjects with Hypertension in China. Kidney Blood Press Res 2017;42:267-275.

19 Levi-Marpillat N, Macquin-Mavier I, Tropeano AI, Parati G, Maison P: Antihypertensive drug classes have different effects on short-term blood pressure variability in essential hypertension. Hypertens Res 2014;37:585-590.

20 Shigenaga A, Tamura K, Dejima T, Ozawa M, Wakui H, Masuda S, Azuma K, Tsurumi-Ikeya Y, Mitsuhashi H, Okano Y, Kokuho T, Sugano T, Ishigami T, Toya Y, Uchino K, Tokita Y, Umemura S: Effects of angiotensin II type 1 receptor blocker on blood pressure variability and cardiovascular remodeling in hypertensive patients on chronic peritoneal dialysis. Nephron Clin Pract 2009;112:c31-40.

-21 Mulè G, Calcaterra I, Costanzo M, Morreale M, D’Ignoto F, Castiglia A, Geraci G, Rabbiolo G, Vaccaro F, Cottone S: Average real variability of 24-h systolic blood pressure is associated with microalbuminuria in patients with primary hypertension. J Hum Hypertens 2016;30:164-170.

22 Feng Y, Li Z, Liu J, Sun F, Ma L, Shen Y, Yilun Z: Association of short-term blood pressure variability with cardiovascular mortality among incident hemodialysis patients. Renal Failure 2018;40:259-264.

-23 Umemoto S, Ogihara T, Matsuzaki M, Rakugi H, Ohashi Y, Saruta T; Combination Therapy of Hypertension to Prevent Cardiovascular Events (COPE) Trial Group: Effects of calcium channel blocker-based combinations on intra-individual blood pressure variability: post hoc analysis of the COPE trial. Hypertens Res 2016;39:46-53.

-24 Wang JG, Yan P, Jeffers BW: Effects of amlodipine and other classes of antihypertensive drugs on long-term blood pressure variability: evidence from randomized controlled trials. J Am Soc Hypertens 2014;8:340349.

25 Yao K, Nagashima K, Miki H: Pharmacological, pharmacokinetic, and clinical properties of benidipine hydrochloride, a novel, long-acting calcium channel blocker. J Pharmacol Sci 2006;100:243-261.

-26 Pico JC, Domínguez G, Negri AL, Caubet JC, Terragno NA: Comparative pharmacokinetics of a single oral dose of two formulations of amlodipine. A randomized, single-blind, two-period, two-sequence, crossover study. Arzneimittelforschung 2008;58:323-327.

27 Zhang Y, Agnoletti D, Safar ME, Blacher J: Effect of antihypertensive agents on blood pressure variability: the Natrilix SR versus candesartan and amlodipine in the reduction of systolic blood pressure in hypertensive patients (X-CELLENT) study. Hypertension 2011;58:155-160.

28 Martre H, Sari R, Taburet AM, Jacobs C, Singlas E: Haemodialysis does not affect the pharmacokinetics of nifedipine. Br J Clin Pharmacol 1985;20:155-158.

-29 Kojima M, Taniguchi M, Sato K, Ueda R, Dohi Y: Antihypertensive effects of long-acting calcium channel blockers on hemodialysis days-a randomized crossover trial between benidipine and nifedipine CR. Nephron Clin Pract 2004;97:c49-53.

-30 Bombelli M, Toso E, Peronio M, Fodri D, Volpe M, Brambilla G, Facchetti R, Sega R, Grassi G, Mancia G: The Pamela study: main findings and perspectives. Curr Hypertens Rep 2013;15:238-243.

-31 Park J, Rhee CM, Sim JJ, Kim Y-L, Ricks J, Streja E, Vashistha T, Tolouian R, Kovesdy CP, Kalantar-Zadeh K: A comparative effectiveness research study of the change in blood pressure during hemodialysis treatment and survival. Kidney Int 2013;84:795-802.

-32 Mena LJ, Maestre GE, Hansen TW, Thijs L, Liu Y, Boggia J, Li Y, Kikuya M, Björklund-Bodegård K, Ohkubo T, Jeppesen J, Torp-Pedersen C, Dolan E, Kuznetsova T, Stolarz-Skrzypek K, Tikhonoff V, Malyutina S, Casiglia E, Nikitin Y, Lind L, et al.: How Many Measurements Are Needed to Estimate Blood Pressure Variability Without Loss of Prognostic Information? Am J Hypertens 2014;27:46-55. 